\title{
Erratum:
}

(Computational and Applied Mathematics, Vol. 23, N. 2-3, pp. 259-284)

\section{Size exclusion during particle suspension transport in porous media: stochastic and averaged equations}

\author{
A. SANTOS $^{1}$ and P. BEDRIKOVETSKY ${ }^{2}$ \\ ${ }^{1}$ Universidade Católica do Rio de Janeiro (PUC-Rio/DEC/GTEP) \\ Rua Marques de São Vicente 225, 22453-900 Rio de Janeiro, RJ, Brazil \\ ${ }^{2}$ Universidade Estadual do Norte Fluminense (UENF/LENEP) \\ Rod. Amaral Peixoto, km 163 - Av. Brenand s/n. \\ 27925-310 Macaé, RJ, Brazil \\ E-mails: asantos@civ.puc-rio.br/pavel@lenep.uenf.br
}

\begin{abstract}
A pore scale population balance model is formulated for deep bed filtration of stable particulate suspensions in porous media. The particle capture from the suspension by the rock occurs by the size exclusion mechanism. The equations for particle and pore size distributions have been derived from the stochastic Master equation. The model proposed is a generalization of stochastic Sharma-Yortsos equations - it accounts for particle flux reduction due to restriction for large particles to move via small pores. Analytical solution for low particle concentration is obtained for any particle and pore size distributions. The averaged macro scale equations, derived from the stochastic pore scale model, significantly differ from the traditional deep bed filtration model.
\end{abstract}

Mathematical subject classification: $76 \mathrm{~S} 05,60 \mathrm{H} 15$.

Key words: deep bed filtration, particle size distribution, pore size distribution, size exclusion mechanism, stochastic model, averaging.

\#566/03. Received: 31/III/03. Accepted: 24/XI/04. 


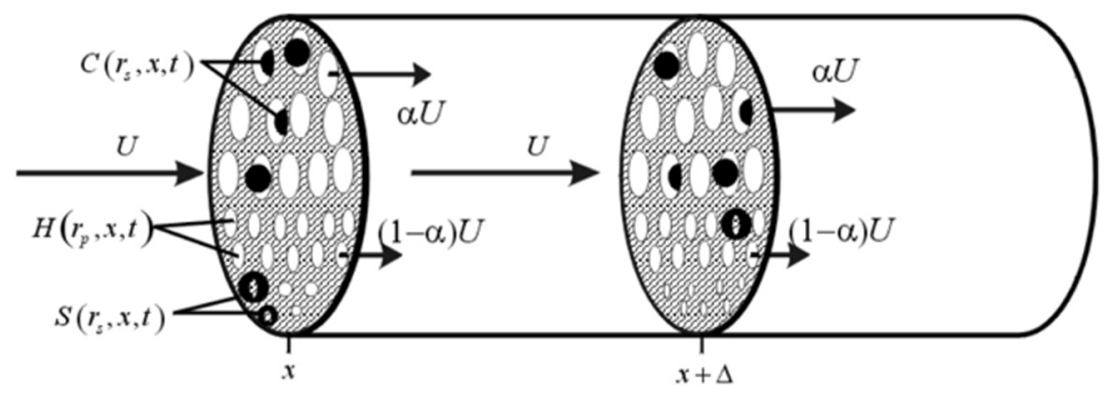

(a)

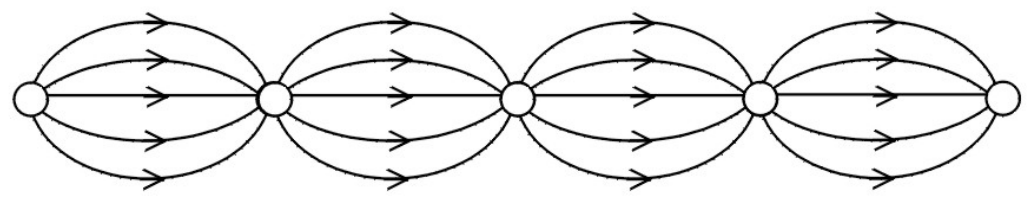

(b)

Figure 1 - Geometric model of the porous medium (a) Sketch for particulate suspension transport in porous media: particles are captured due to size exclusion. (b) Connectivity in the porous medium model.

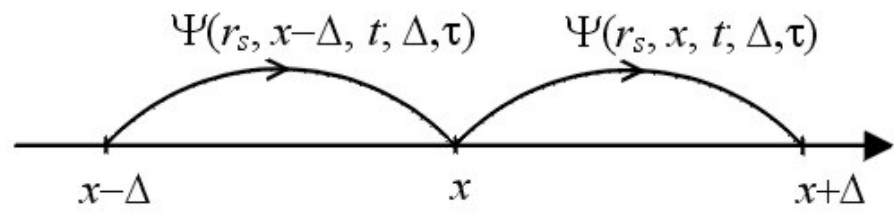

Figure 2 - Transition probabilities for particle displacement in Master equation.

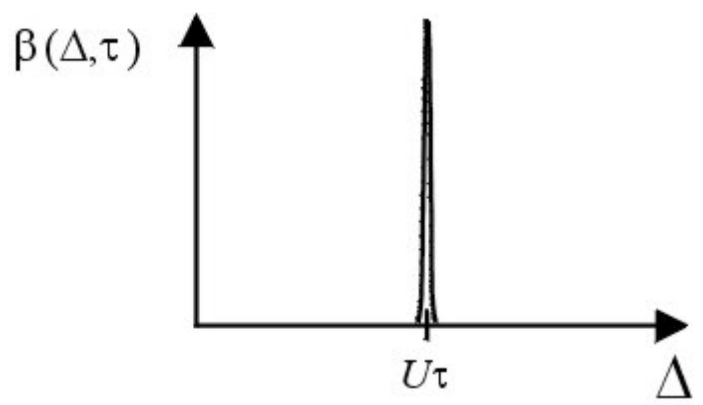

Figure 3 - Shape of the fluid displacement probability function $\beta$. 


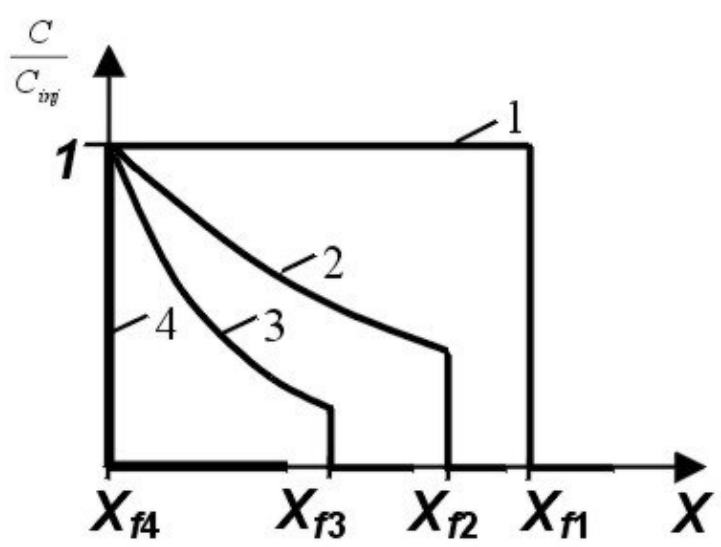

Figure 4 - Concentration waves for different particle sizes. Each front moves with velocity $\alpha\left(r_{s}\right)$. Line 1 corresponds to transport of small particles $\left(r_{s 1}<r_{p m i n}\right)$. Lines 2 and 3 are related to filtration of intermediate size particles, $r_{s 2}<r_{s 3}$. Line 4 shows the large particle concentration $\left(r_{s 4}>r_{p \max }\right)$.

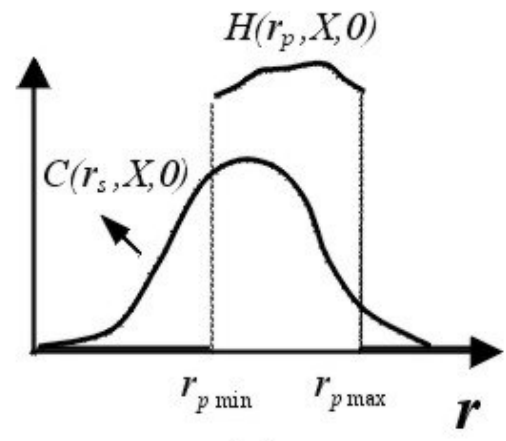

(a)

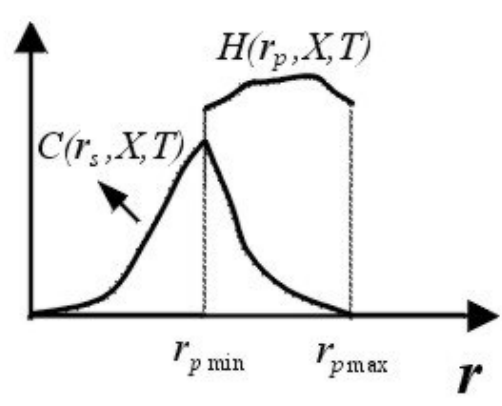

(b)

Figure 5 - Concentration distributions for suspended particles and vacant pores during filtration: (a) initial distributions; (b) distributions behind the concentration front for $T>0$. 


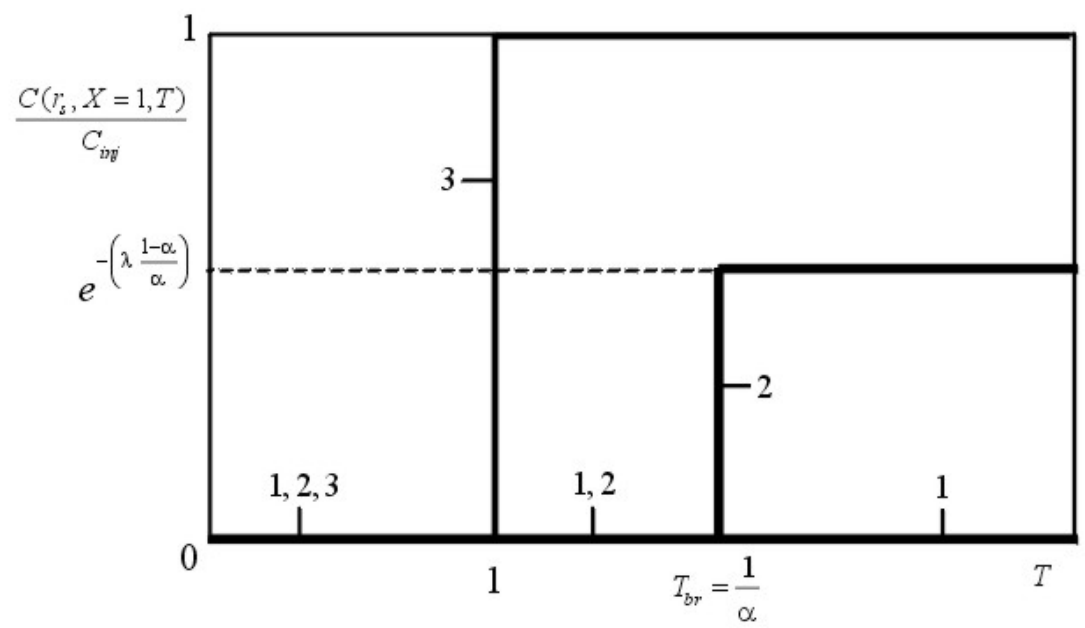

Figure 6 - Suspended concentration at the core outlet. Lines 1 and 3 are related to large and small particles, respectively. Line 2 corresponds to intermediate size particle concentration.

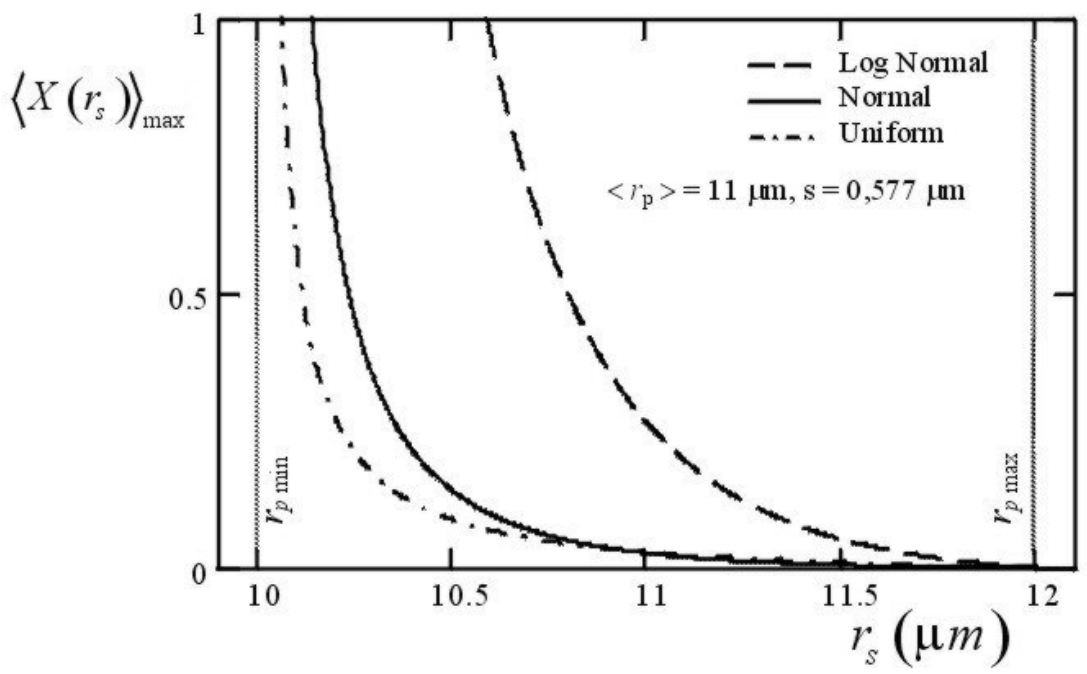

Figure 7 - Effect of particle size on dimensionless penetration depth $\langle X(r s)\rangle_{\max }$ for intermediate size particles. Three curves were calculated for three different pore size distributions assuming the same average pore size $(\langle r p\rangle=11 \mu \mathrm{m})$, standard deviation $(s=0 ; 577 \mu m)$ and dimensionless filtration coefficient $(\lambda=50)$. 


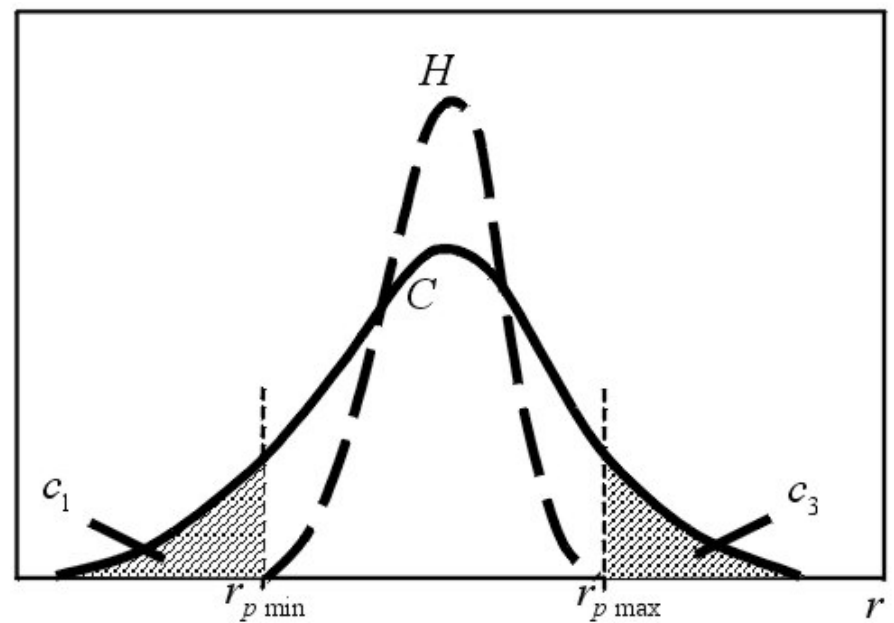

Figure 8 - Shapes of suspended particle and vacant pore size distributions. Areas $c_{1}$ and $c_{3}$ represent the fraction of particles that do not perform deep bed filtration (small and large particles, respectively). 